\title{
Etomidate, but not thiopental, decreases serum cortisol concentration in morbidly obese patients. A randomized controlled trial
}

\author{
Marcin Możański ${ }^{1}$, Dariusz Tomaszewski ${ }^{1}$, Zbigniew Rybicki ${ }^{1}$, Jolanta Bejm², Mariusz Bałkota ${ }^{1}$ \\ ${ }^{1}$ Department of Anesthesiology and Intensive Care, Military Institute of Medicine, Warsaw, Poland \\ ${ }^{2}$ Department of Laboratory Diagnostics, Military Institute of Medicine, Warsaw, Poland
}

\begin{abstract}
Background: The effect of etomidate administration on the adrenal cortex in obese patients is still unclear. The objective of the study was to determine the influence of a single dose of etomidate on cortisol secretion in the morbidly obese.

Methods: 127 healthy patients were enrolled into the study. Data from 82 patients scheduled for elective laparoscopic surgery were analyzed. 62 of them were morbidly obese, while 20 had normal body mass. The participants were divided equally into etomidate and thiopental groups, depending on the kind of intravenous anaesthetic used for the induction of anaesthesia. Each patient's serum cortisol concentration was measured five times: on the day before surgery (sample A), two hours after the induction of anaesthesia (sample B), after a short tetracosactide test (sample C), 24 hours after the induction of anaesthesia (sample D), and after a second short stimulation test (sample E). Results: The mean cortisol concentration in obese patients in the etomidate group was lower two hours after the induction of anaesthesia (sample $B, P<0.001$ ), and 30 minutes after the first tetracosactide test (sample $C, P<0.001$ ) compared to obese patients in the thiopental group. There were no differences between the groups in sample $A$ $(P=0.833), \mathrm{D}(P=0.614)$ and $\mathrm{E}(P=0.769)$. We found no changes in haemodynamic parameters between both groups. Conclusions: Etomidate decreased serum cortisol concentration and decreased reactivity to tetracosactide both in morbidly obese and in normal weight patients. This effect was reversible within 24 hours.
\end{abstract}

Key words: anaesthetics, intravenous, etomidate; anaesthetics, intravenous, thiopental; obesity, morbid; hormones, serum cortisol

Obesity is becoming an increasing social problem. In $2008,33.8 \%$ of the US adult population was obese (BMI > $30 \mathrm{~kg} \mathrm{~m}^{-2}$ ), and the medical costs of treatment of obesity were estimated to be 147 billion US dollars. The number of both surgical and anaesthetic procedures performed in obese patients has been increasing. Etomidate is one of the intravenous anaesthetics used in obese patients because of its mild circulatory effect and the prompt return of consciousness [1]. However, etomidate can inhibit the activity of the CYP11B1 isoenzyme in the adrenal cortex, resulting in decreased concentrations of cortisol, cortisone and aldosterone, as well as increased concentrations of 11-deoxycorticosterone, 11-deoxycortisole, progesterone and 17-hydroxyprogesterone [2]. After administration of a single dose of etomidate, the inhibitory effect can persist up to 6-8 hours [3], and - in cases of intravenous infusion up to 24 hours [4]. As there have been some clinical reports of increased mortality related to the intravenous administration of etomidate in critically ill individuals [5], currently, the only approved way of administration of etomidate is by a single intravenous injection.

There is a relationship between abdominal obesity and changes of the hypothalamic-pituitary-adrenal axis [6]. Moreover, although the role of cortisol as a mediator in 
idiopathic obesity has been suggested, the serum cortisol concentrations in obese patients were not_increased. The tissue-specific response to cortisol may be the outcome of the circulating concentration of this hormone, as well as prereceptor metabolism and changes in $11 \beta$-hydroxysteroid dehydrogenase type 1 (11ß-HSD1) activity [7]. In concert, these abnormalities seem to be responsible for the abnormal metabolism often seen in central obesity [8].

The effects of adrenal cortex suppression are much more visible in obese patients than in those with normal body weight. Circulatory stability is lower, wound healing is slower, and muscular weakness is increased. It can also affect the course of the postoperative period: both hospitalization time may be prolonged and the costs of medical procedures may be increased. We did not find any studies in the current medical literature on the effects of etomidate administration on the adrenal cortex in obese patients.

The objective of the study was: to analyze the effect of etomidate on cortisol secretion in obese patients under general anaesthesia.

\section{METHODS}

The study was planned as a randomized controlled trial. After the commencement of the study its design was not modified. The study was registered in the Clinical Trials database (NCT01930019).

After Ethics Committee approval, written informed consent was obtained from each subject. Patients in ASA (American Society of Anaesthesiologists) class I and II awaiting elective abdominal laparoscopic surgery were included in the study. The patients excluded from the study were those who: 1) did not agree to participate, 2) were being treated with steroidal drugs, 3) had cortisol metabolism disorders or were being treated with drugs with a potential impact on serum cortisol concentration, 4. had a preoperative risk assessment result of ASA class above II, 5) had had an initial surgical laparoscopic technique converted to a laparotomy, and 6. had had surgical complications increasing the level of intraoperative stress.

One hour before the induction of general anesthesia in all the patients, $7.5 \mathrm{mg}$ of midazolam (Dormicum, Egis Pharmaceuticals Ltd, Budapest, Hungary) was administered orally as a premedication.

After the patient's arrival in the operating theatre, standard monitoring including continuous electrocardiography, noninvasive blood pressure measurement and pulsoximetry was commenced. An intravenous cannula was inserted and a crystalloid infusion started. All the patients were oxygenated. Depending on the study group, either etomidate or thiopental was used as an induction agent for general anaesthesia. Both anaesthetics were administered according to ideal body weight. The dose of etomidate (Etomidate lipuro,
B.Braun Melsungen, Germany) varied from 0.15 to $0.3 \mathrm{mg} \mathrm{kg}^{-1}$. The dosage of thiopental (Thiopental Sandoz GmbH, Kundl, Austria) varied from 2 to $5 \mathrm{mg} \mathrm{kg}^{-1}$. When the ciliary reflex disappeared, either suxamethonium chloride (Chlorsuccilin, Przedsiębiorstwo Farmaceutyczne Jelfa, Jelenia Góra, Poland) or rocuronium bromide (Esmeron, N.V.Organon, Oss, the Netherlands) was administered, depending on the possible problems in endotracheal intubation. General anaesthesia was maintained with desflurane (Suprane, Baxter Poland), continuous intravenous infusion of ultra-short acting opioid remifentanil (Ultiva, GlaxoSmithKline Manufacturing S.p.A. San Polo di Torrile, Italy), and repeated doses of rocuronium bromide as required. Both inspiratory and expiratory oxygen concentration, end-tidal concentration of carbon dioxide, as well as inspiratory and expiratory concentrations of volatile anaesthetic were measured intraoperatively.

Each patient had his or her serum cortisol concentration level measured five times. The first measurement was taken on the afternoon of the day before surgery (A sample), and the second two hours after the induction of anaesthesia with etomidate or thiopental (B sample). Subsequently a short stimulation test with intravenous injection of $250 \mu \mathrm{g}$ of tetracosactide (Synacthen, Sigma-tau GmbH, Düsseldorf, Germany), a synthetic analogue of adrenocorticotropic hormone (ACTH), was performed. The third sample was taken thirty minutes after the short stimulation blood test (C sample), and subsequently, the fourth (D) sample was taken 24 hours after the induction of anaesthesia. Immediately afterwards, a second short stimulation test with $250 \mu \mathrm{g}$ of tetracosactide was performed, and 30 minutes later, the fifth blood sample (E sample) was taken.

The serum cortisol concentration was measured by electrochemiluminescence (ECL) with a Cobas e411 analyzer (Roche Diagnostics GmbH, Germany).

The patients' participation of the study ended when the final, fifth blood sample was taken.

The participants were subsequently randomly and divided into etomidate and thiopental groups, according to the intravenous anaesthetic used as the induction agent for general anesthesia. Finally, four groups of patients were analyzed: 1. obese, in which etomidate was used (OE group),

2. obese, in which thiopental was used (OT group).

All the patients were included, and randomly divided, into groups by the authors of the study. The study was not blinded.

\section{STATISTICAL ANALYSIS}

Collected data were archived with Microsoft Office 2010 software. The statistical analysis was performed using $\mathrm{R}$ statistical package, version 3.1.2. ${ }^{1}$

Descriptive statistics were used to summarize the sample results. 


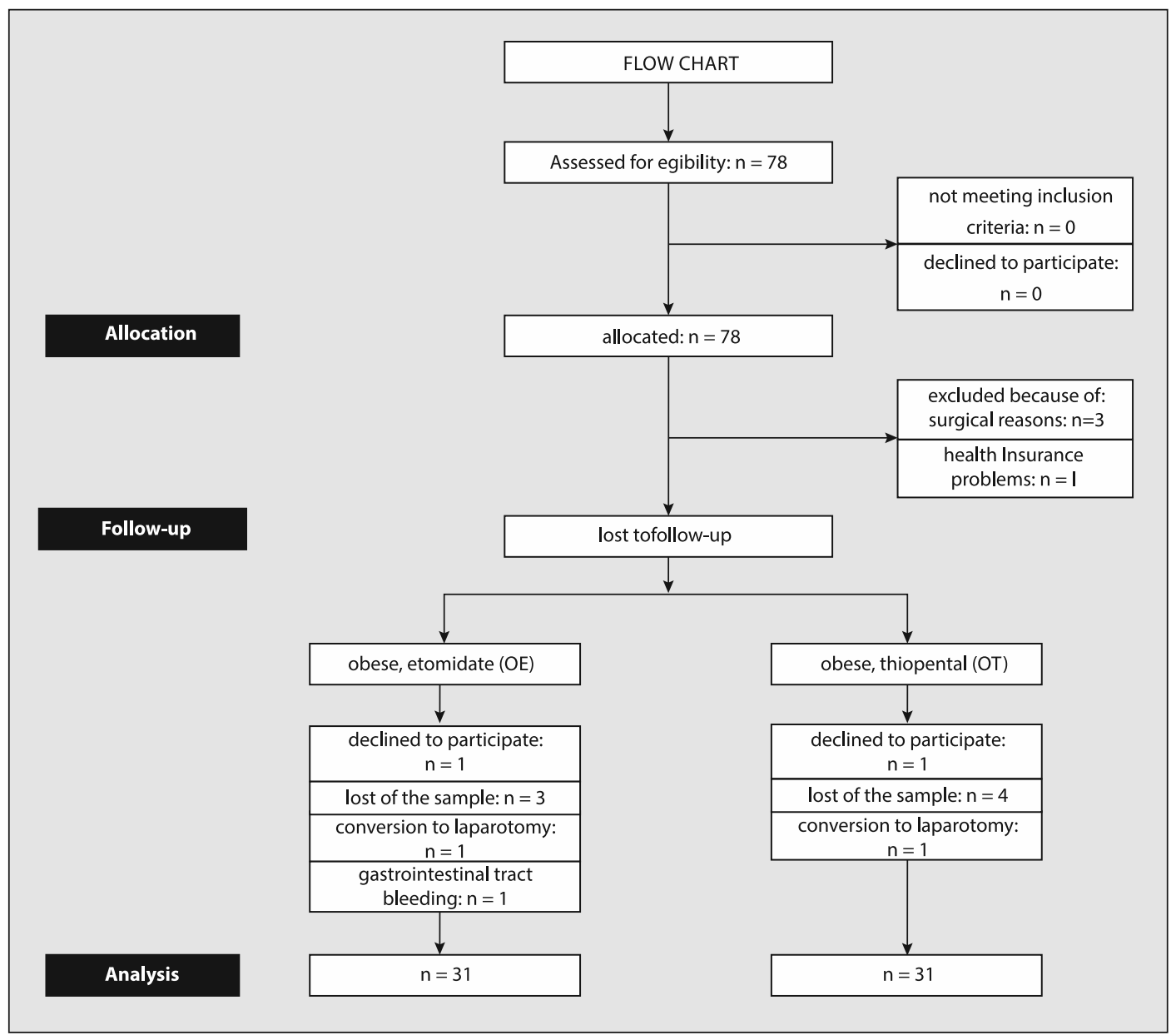

Figure 1. Flow chart

The chi-squared and Student t tests were used to analyze the differences in sex and age between groups.

Chi squared test was used to analyze the differences in nominal data between groups. The Kolmogorov-Smirnov, Lilliefors and Shapiro-Wilk tests were used to analyze normality in of the distribution of all remaining data. If analyzed data passed the normality test, the analysis of variance with Levene and Brown-Forsythe tests were performed. When the variances were equal, the differences between groups were analyzed with Student t-test. The differences between groups regarding data violating normality assumption were analyzed with Mann-Whitney $U$ test.

$P<0.05$ was considered statistically significant.

\section{RESULTS}

The participants' flow in the study is summarized in Figure 1. The demographic data of the participants is shown in Table 1.
There were no differences in number of men and women between study and control group ( $P=0.43$ ). There were no differences between both groups in terms of age, body weight and BMI ( $P$ values $0.52,0.44$ and 0.195, respectively).

The final data collected from 62 patients were analyzed. The surgical bariatric procedures in obese patients (OE and OT groups, 31 patients each) included: 49

Table 1. Demographic data of the participants

\begin{tabular}{lccc}
\hline & Group & $\begin{array}{c}\text { Mean/ } \\
\text { /median }\end{array}$ & $\begin{array}{c}95 \% \text { Cl for mean/ } \\
\text { /median }\end{array}$ \\
\hline Age [years] & 1 & 42 & $38-47$ \\
Body weight $[\mathrm{kg}]\left(^{*}\right)$ & 1 & 144 & $37-45$ \\
& 2 & 135 & $137-152$ \\
BMI [kg m ${ }^{-2}$ ] & 1 & 49.9 & $47.2-52.6$ \\
& 2 & 47.5 & $45.1-50.0$ \\
\hline
\end{tabular}

$\left({ }^{*}\right)$ data violating normality assumption 
Table 2. Summary of analyzed data - cortisol concentrations

\begin{tabular}{lccc}
\hline & Group & $\begin{array}{c}\text { Mean/ } \\
\text { /median }\end{array}$ & $\begin{array}{c}95 \% \mathrm{Cl} \\
\text { for mean/ } \\
\text { /median }\end{array}$ \\
\hline Sample A & 1 & 10.53 & $8.65-12.41$ \\
Sample B $\left.{ }^{*}\right)$ & 2 & 10.83 & $8.89-12.77$ \\
Sample C $\left.{ }^{*}\right)$ & 1 & 11.01 & $8.7-14.9$ \\
Sample D & 2 & 26.90 & $23.7-32.1$ \\
& 1 & 13.60 & $11.5-15.8$ \\
Sample E $\left.{ }^{*}\right)$ & 1 & 33.80 & $32.8-38.3$ \\
& 2 & 28.92 & $24.59-33.25$ \\
& 1 & 26.21 & $21.33-31.20$ \\
\hline
\end{tabular}

$\left(^{*}\right)$ data violating normality assumption

sleeve gastrectomies ( 27 in the OE group and 22 in the OT group), 8 adjustable gastric banding (ASGB) procedures ( 2 in the OE and 6 in the OT group), and 5 Roux-en-Y gastric bypass (RYGB) procedures ( 2 in the OE group and 3 in the OT group).

The mean time of a surgical procedure was 125 minutes.

The results of serum cortisol measurements are shown in Table 2.

One day before surgery the mean serum concentration of cortisol (A sample) in the etomidate groups was: 10.53 $\pm 5.13 \mu \mathrm{g} \mathrm{dL}^{-1}$. In thiopental groups the mean serum concentration of cortisol was $10.83 \pm 5.29 \mu \mathrm{g} \mathrm{dL}^{-1}$. Those values were within the normal range.

Two hours after etomidate administration (B sample) the serum cortisol concentration was $12.80 \pm 7.40 \mathrm{\mu g} \mathrm{dL}^{-1}$.

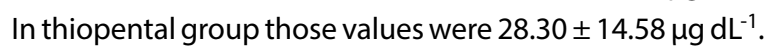

After the short stimulation test (C sample), the mean serum concentration of cortisol in the etomidate group was $14.99 \pm 6.63 \mu \mathrm{g} \mathrm{dL}^{-1}$. In the thiopental group this values was $36.28 \pm 10.16 \mu \mathrm{g} \mathrm{dL}^{-1}$.

Twenty-four hours after induction of anesthesia the mean serum concentration of cortisol ( $D$ sample) in the etomidate group was $28.92 \pm 11.80 \mathrm{\mu g} \mathrm{dL}^{-1}$. In the thiopental group it was $26.21 \pm 13.58 \mathrm{\mu g} \mathrm{dL}^{-1}$.

After the short stimulation test the mean serum concentrations of cortisol was $39.82 \pm 10.24 \mathrm{~g} \mathrm{dL}^{-1}$ (in OE group), $34.31 \pm 9.94 \mathrm{mg} \mathrm{dL}^{-1}$ (in OT group).

The summary of these results was shown in Table 2.

In the thiopental group (OT) the mean serum concentration of cortisol was higher (1) two hours after induction of anesthesia (B sample), and (2) 30 minutes after first administration of tetracosactide (C sample) compared to patients from the etomidate group (OE).
We found no such relation for samples collected (1) the day before surgery (A sample), (2) 24 hours after induction of anesthesia ( $D$ sample), and (3) 30 minutes after second administration of tetracosactide (E sample).

The comparison of mean cortisol concentration in obese patients between OE and OT groups revealed that significant differences were related to (1) the B sample, collected 2 hours after induction of anesthesia, and (2) the $C$ sample, collected 30 minutes after first administration of tetracosactide. There were no differences between the $A, D$ and E sample. The $P$ values were 0.821 (A sample),$<0.001$ (B sample), < 0.001 (C sample), 0.405 (D sample) and 0.767 (E sample), respectively.

In addition, hemodynamic parameters (blood pressure, heart rate) in each group were analyzed.

The summary of the results is shown in Table 3.

We found no changes in hemodynamic parameters between etomidate and thiopental groups.

Analysis of heart rate changes showed that $P$ values in obese patients were 0.125 for heart rate increases, and 0.805 for heart rate decreases. Analysis of intraoperative changes of blood pressure showed that p values in obese patients were 0.683 for blood pressure increases and 0.559 for blood pressure decreases.

None of the participants had adverse reactions, which could be associated with the protocol of the study.

\section{DISCUSSION}

The issue of serum cortisol concentration in obese patients is still unclear. Some authors have observed central adrenal insufficiency with concurrent disorders of insulin and lipoprotein metabolism in this group [9]. Others have reported that both $\mathrm{ACTH}$ and cortisol concentration may be increased $[10,11]$. Although some data suggest serum cortisol concentration and circadian rhythm of cortisol secretion may be normal in this group, production of this hormone, as well as its degradation, were increased [8] — a state of functional hypercortisolism [12]. Our results suggest that initial cortisol concentration in obese patients, are within normal range. This is an important data point, especially for patients awaiting surgical procedures. Etomidate is often used as an induction agent for general anaesthesia in this group of patients. The inhibition of steroidogenesis caused by etomidate has been known for many years. We confirmed this in our study in obese patients. The increase in serum cortisol concentration after intravenous administration of tetracosactide, two hours after the induction of anaesthesia with etomidate, was less than $7 \mu \mathrm{g} \mathrm{dL}^{-1}$, while in the thiopental group, the results of the stimulation test indicated normal functioning of the adrenal glands. These results are consistent with those of other authors. The decreased serum cortisol concentration after intravenous administration of 
Table 3. Hemodynamic parameters

\begin{tabular}{lccc}
\hline & Group & $\begin{array}{c}\text { Mean/ } \\
\text { /median }\end{array}$ & $\begin{array}{c}95 \% \mathrm{Cl} \text { for } \\
\text { mean/median }\end{array}$ \\
\hline Heart rate increase $\left(^{*}\right)$ & 1 & 14.20 & $5.55-22.2$ \\
Heart rate decrease & 2 & 5.80 & $0.00-12.5$ \\
& 1 & 21.91 & $17.89-25.92$ \\
Blood pressure & 1 & 22.68 & $17.67-27.70$ \\
increase $\left(^{*}\right)$ & 2 & 0.00 & $0.00-3.00$ \\
Blood pressure & 1 & 0.00 & $0.00-2.10$ \\
decrease & 2 & 37.66 & $34.31-41.01$ \\
Duration of the & 1 & 130 & $31.68-40.46$ \\
surgical procedure $\left({ }^{*}\right)$ & 2 & 105 & $90-120-150$ \\
\hline
\end{tabular}

$\left.{ }^{*}\right)$ data violating normality assumption

etomidate is due to the reversible inhibition of 11-betahydroxylase $[8,12]$, although in our study, the activity of this enzyme was not measured.

Our results show that etomidate-induced adrenal suppression takes no more than 24 hours. The mean serum cortisol concentration in obese patients was similar, both in the etomidate and the thiopental group. These observations were confirmed by the results of the short stimulation test performed twenty-four hours after the induction of general anaesthesia. The estimated time of adrenal suppression caused by a single dose of etomidate varies between 12 hours [13] and 48 hours [14]. Our results were within this range.

The dosage of anaesthetics used as induction agents for general anaesthesia were calculated according to ideal body weight. However, the doses of anaesthetics used were verified clinically. The dose of etomidate varied from 0.15 to $0.3 \mathrm{mg} \mathrm{kg}^{-1}$ body weight. Although the hypnotic serum concentration of etomidate is about $200 \mathrm{ng} \mathrm{mL}^{-1}$, its inhibitory effect on steroidogenesis is observed at $10 \mathrm{ng} \mathrm{mL}^{-1}$ [1]. The current literature does not answer the question whether the inhibition of steroidogenesis is dose-dependent, or whether there is a kind of a "ceiling effect". Thus, it is difficult to draw conclusive clinical conclusions.

One of the biggest advantages of etomidate is its minimal cardiovascular effects. However, it is not an ideal anaesthetic. The higher incidence of adrenal insufficiency and increased mortality after etomidate administration for the rapid induction of anaesthesia has been shown in septic patients [15]. Indeed, both decreased cortisol secretion after stimulation with ACTH and increased mortality during a 28-day period was shown by Cuthbertson et al. [16] in his analysis of 499 critically ill patients. Even single doses of etomidate can facilitate the progress of inflammatory changes in the lungs of traumatic patients [17]. Such scenarios in patients with obesity could be extremely dangerous.

In our study transient adrenal insufficiency was observed after intravenous administration of etomidate. This did not last long and, taking into account the relatively short observation, we did not observe any important clinical implications. None of our patients had prolonged neuromuscular blockade, although proper cortisol concentration is necessary to correct the functioning of the neuromuscular joint [18]. Moreover, we did not find significant differences in hypotension episodes. However, only relatively healthy patients, with preoperative ASA status I and II were included in this study.

There is no clear answer as to the safety of etomidate administration. Chan et al. in their metanalysis of papers between January 1950 and February 2012 [19] showed both an increased incidence of adrenal insufficiency and mortality in patients who received a single dose of etomidate prior to rapid intubation. Apart from the fact that etomidate had been introduced as an anaesthetic agent in 1972 in Europe and in 1983 in the United States, McPhee et al. [20] in their retrospective cohort study of 741,036 patients hospitalized between 2008 and 2010, found no correlation of etomidate administration in rapid sequence intubation and increased mortality and other complications.

It is quite difficult to apply the results found in critically ill patients to those with morbid obesity. Our results confirmed the inhibitory effect of etomidate on the synthesis of cortisol, extending these observations to the morbidly obese. Further research is necessary for a more accurate evaluation of the clinical consequences of these observations.

\section{ACKNOWLEDGEMENTS}

1. Source of funding: own sources of Military Institute of Medicine.

2. The authors declare no conflict of interest.

\section{References:}

1. Forman SA: Clinical and molecular pharmacology of etomidate. Anesthesiology 2011; 114: 695-707. doi: 10.1097/ ALN.0b013e3181ff72b5.

2. Dörr HG, Kuhnle U, Holthausen H, Bidlingmaier F, Knorr D: Etomidate: a selective adrenocortical 11 beta-hydroxylase inhibitor. Klin Wochenschr 1984; 62: 1011-1013.

3. Allolio B, Stuttmann R, Leonhard U, Fischer H, Winkelmann W: Adrenocortical suppression by a single induction dose of etomidate. Klin Wochenschr 1984; 62: 1014-1017.

4. Wanscher M, Tønnesen E, Hüttel M, Larsen $K$ : Etomidate infusion and adrenocortical function. A study in elective surgery. Acta Anaesthesiol Scand 1985; 29: 483-485.

5. Ledingham IM, Watt I: Influence of sedation on mortality in critically ill multiple trauma patients. Lancet 1983; 1: 1270.

6. Rosmond R, Dallman MF, Björntorp P: Stress-related cortisol secretion in men: relationships with abdominal obesity and endocrine, metabolic and hemodynamic abnormalities. J Clin Endocrinol Metab 1998; 83: 1853-1859. 
7. RaskE, Olsson T, Söderberg S et al.: Tissue-specific dysregulation of cortisol metabolism in human obesity. J Clin Endocrinol Metab 2001; 86: 1418-1421.

8. Björntorp P, Rosmond R: Obesity and cortisol. Nutrition 2000; 16: 924-936.

9. Hautanen A, Adlercreutz H: Altered adrenocorticotropin and cortisol secretion in abdominal obesity: Implications for the insulin resistance syndrome. J Intern Med 1993; 234: 461-469.

10. Walker BR: Cortisol-cause and cure for metabolic syndrome? Diabet Med 2006; 23: 1281-1288.

11. Sen $Y$, Aygun D, Yilmaz E, Ayar A: Children and adolescents with obesity and the metabolic syndrome have high circulating cortisol levels. Neuro Endocrinol Lett 2008; 29: 141-145 .

12. Lordelo RA, Mancini MC, Cercato C, Halpern A: Hormonal axes in obesity: Cause or effect? Arq Bras Endocrinol Metabol 2007; 51: 34-41.

13. Schenarts $C L$, Burton $J H$, Riker RR: Adrenocortical dysfunction following etomidate induction in emergency department patients. Acad Emerg Med 2001; 8: 1-7.

14. Archambault P, Dionne CE, Lortie G, LeBlanc F, Rioux A, Larouche $G$ : Adrenal inhibition following a single dose of etomidate in intubated traumatic brain injury victims. CJEM 2012; 14:270-282.

15. Albert SG, Ariyan S, Rather A:The effect of etomidate on adrenal function in critical illness: A systematic review. Intensive Care Med 2011; 37: 901-910. doi: 10.1007/s00134-011-2160-1.
16. Cuthbertson BH, Sprung CL, Annane D et al:: The effects of etomidate on adrenal responsiveness and mortality in patients with septic shock. Intensive Care Med 2009; 35: 1868-1876. doi: 10.1007/s00134-009-1603-4.

17. Asehnoune $K$, Mahe PJ, Seguin P et al.: Etomidate increases susceptibility to pneumonia in trauma patients. Intensive Care Med 2012; 38: 1673-1682.

18. Alvarez A, Bordsky JB, Lemmens HJ, Morton JM: Morbid obesity Perioperative management. Oxford University Press 2010.

19. Chan CM, Mitchell $A L$, Shorr AF: Etomidate is associated with mortality and adrenal insufficiency in sepsis: a metaanalysis. Crit Care Med 2012; 40: 2945-2953. doi: 10.1097/ CCM.0b013e31825fec26.

20. McPhee $L C$, Badawi O, Fraser GL et al.: Single-Dose etomidate is not associated with increased mortality in ICU patients with sepsis: Analysis of a large electronic ICU database. Crit Care Med 2013; 41: 774-783. doi: 10.1097/CCM.0b013e318274190d.

\section{Corresponding author:}

Marcin Możański

Klinika Anestezjologii i Intensywnej Terapii WIM

ul. Szaserów 128, 04-141 Warszawa, Poland

e-mail:mmozanski@wim.mil.pl

Received: 7.07.2014

Accepted: 30.06 .2015 